\title{
Detecting gallbladders in chicken livers using spectral analysis
}

\author{
Anders Jørgensen ${ }^{1}$ \\ andjor@create.aau.dk \\ Eigil Mølvig Jensen² \\ emj@infood.dk \\ Thomas B. Moeslund ${ }^{1}$ \\ tbm@create.aau.dk
}

\author{
${ }^{1}$ Media Technology \\ Aalborg University \\ Aalborg, DK \\ ${ }^{2}$ IHFood \\ Carsten Niebuhrs Gade 10, 2. tv \\ Copenhagen, DK
}

\begin{abstract}
This paper presents a method for detecting gallbladders attached to chicken livers using spectral imaging. Gallbladders can contaminate good livers, making them unfit for human consumption. A data set consisting of chicken livers with and without gallbladders, has been captured using 33 wavelengths within the visible spectrum. This work shows how to reduce the high number of wavelengths while maintaining a high accuracy. A classification tree has be trained to evaluate if a gallbladder is present and whether it is suitable for automatic removal, which could increase profits for the processing plants. As a preliminary study this shows good results with a classification accuracy of $91.7 \%$.
\end{abstract}

\section{Introduction}

The consumption of chicken meat is increasing in almost every corner of the world. From 2000 to 2011 there has been a $31 \%$ increase in the top chicken producing countries, according to the International Poultry Council[ $[\square]$. To maintain a high quality while increasing the production speed, poultry processing plants relies on computer vision for a larger numbers of inspection tasks. Already existing works can detect skin tumours[ $[\mathbf{G}, \square]$ and faecal matter $[\square, \mathbb{\square}]$ while others sort chickens by wholesome/unwholesome[ [प, $\square]$ ]. Similar methods also exist in commercial systems by Linco and Meyn[ $[$, 四].

But quality inspection of the chicken's organs have received little attention in research. After evisceration, the liver, heart and gizzard are sorted out from the remaining guts. These can be used for sausages, pâté or sold as is. The quality decides whether they are sold for human consumption or are to be used in fodder for animals. And this has a high affect on the price of these organs. Today this quality assessment relies heavily on manual inspection.

Due to the chicken's anatomy, the gallbladder is sometimes by mistake extracted with the liver during evisceration. This means that the liver is unfit for human consumption and thereby only worth $25 \%$ that of a clean liver. It is therefore important to separate livers with and without gallbladders correctly. This paper presents a preliminary study proposing a method for detecting gallbladders among livers using spectral images within the visible spectrum. It further investigates whether detected gallbladders are fit for automatic removal. 


\section{Related Works}

Hyperspectral imaging in relation to food quality and safety receives a growing attention these years, as described by Huang et. al in [0]. Both in superficial inspection of beefmarbling $[\Theta]$, estimating bacteria on the surface $[\square]$ to internal quality like tenderness. Using the NIR spectrum, Elmasry et. al [日] are capable of estimating the $\mathrm{pH}$-value and tenderness of fresh beef. Work by Kamruzzaman et. al [ $[$ ] show that it was also possible to estimate water holding capacity for lamb meat using images captured within the NIR spectrum.

Little research has been done regarding chicken viscera. Tao et. al [Ш]] use ultra violet light to detect splenomegaly in turkey carcasses. The spleen appears almost completely dark in images captured at a central wavelength at $365 \mathrm{~nm}$, making it recognizable from the liver.

Spectroscopy has also been used to detect septicemia in chicken livers[]. Using a neural network they are able to detect $94 \%$ of the septox livers in their sample.

\section{Image Acquisition}

For this study, a data set consisting of 60 image sets was captured off-line at a chicken processing plant. 30 image sets contains just a liver, and 30 image sets contains a liver with a gallbladder attached. The images have been captured using a monochrome Basler Scout camera combined with a VariSpec Liquid Crystal Tunable Filter. The filter has a bandwidth of $10 \mathrm{~nm}$ and a range from $400 \mathrm{~nm}$ to $720 \mathrm{~nm}$, both inclusive. It was chosen to capture images in steps of $10 \mathrm{~nm}$, which results in 33 grey scale images in each image set. The image resolution is $1024 \times 768$. Examples from the data set can be seen in figure 1 .

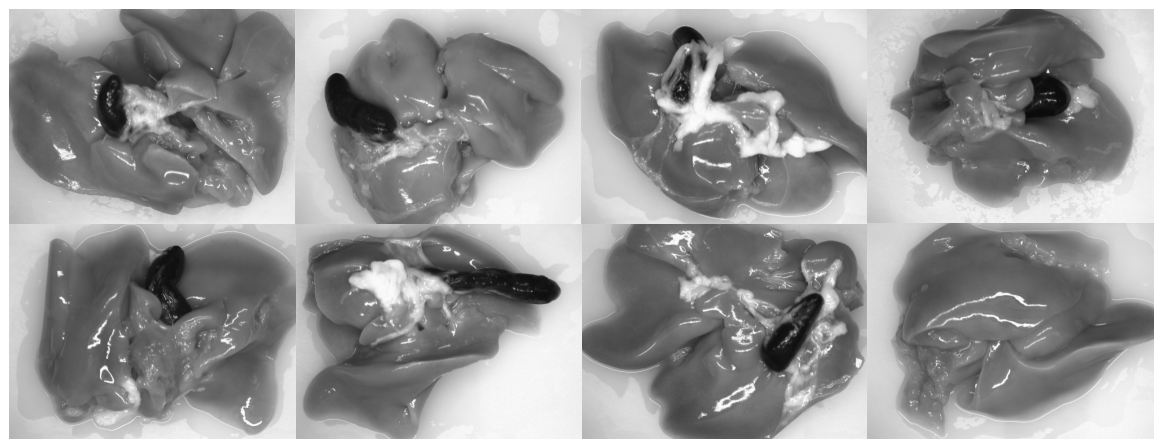

Figure 1: Examples of liver images, captured at $650 \mathrm{~nm}$, from the data set. The black organ is the gallbladder and the white matter is fat.

Before capturing the data set, the lighting was measured using an Ocean Optics spectrometer. This was done at the white region of an X-rite Colour Checker Classic []]. The result can be seen in figure 2 .

To ensure an equal brightness level across the spectrum, the camera's exposure time must be adjusted for each wavelength. High light intensity means a low exposure time. This was done through a calibration routine where the average pixel value for the white region of the colour checker was equalised across all wavelengths. 


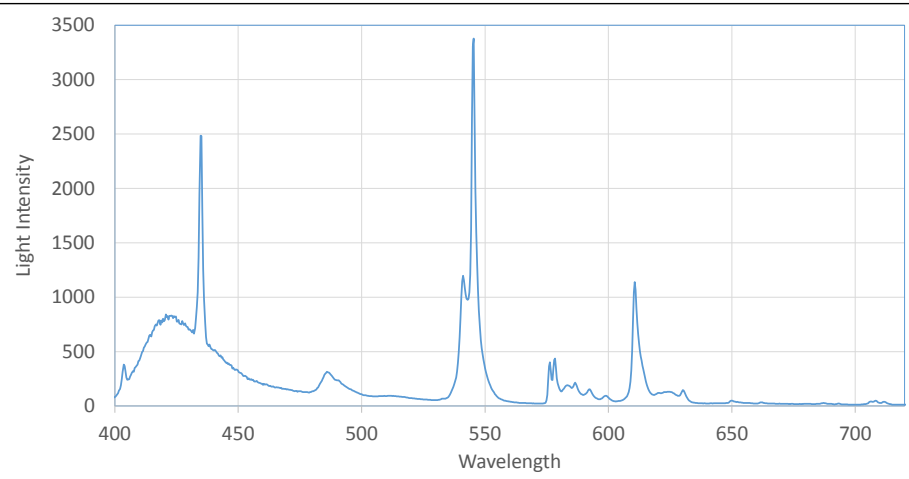

Figure 2: The spectral response of a white surface under the image capture setup.

\section{Methods}

\subsection{Dimensionality Reduction}

The 33 grey scale images are most likely highly correlated, hence many of them can be removed without loss of information. For an on-line system, capturing 33 images at production speeds near 3 birds per second, would also be impractical and expensive. A better solution would be to select the important wavelengths and capture images at those.

For this, Hierarchical Dimensionality Reduction has been used, as this method finds the most uncorrelated subset of already existing features[ [प]]. Pixels extracted from annotated images, containing the three classes "liver", "fat" and "gall", were concatenated in a $n \times 33$ matrix, where 33 is the number of wavelengths. $n$ is the total of 85,352 gall pixels, 321,193 liver pixels and 47,515 fat pixels.

First a classification tree was trained using 10 fold cross validation to find the accuracy when using all wavelengths. The tree was set to contain a maximum of 100 nodes. Then, using the correlation matrix, the two most correlated wavelengths are selected. The Shannon entropy is calculated for both wavelengths and the one with the lowest entropy is then removed from the data set. The decision tree is then trained again. These steps are repeated until the accuracy of the classification tree starts to fall. The results can be seen in figure 3 .

The accuracy is $\approx 0.999$ until one wavelength remains, where it drops to 0.964 . It is therefore chosen to use two wavelengths, to maintain a high accuracy. The two remaining wavelengths before the accuracy drops are $600 \mathrm{~nm}$ and $720 \mathrm{~nm}$. The resulting intensity space can be seen in figure 4 . This shows that the three classes can be separated using these two wavelengths.

\subsection{Segmenting the images}

Using the decision tree trained during the dimensionality reduction, segmenting the gallbladder is straight forward. Every image is smoothed with a $5 x 5$ median filter to removed pixel outliers and then segmented by running each pixel through the tree where it is classified as either "liver", "fat" or "gall". Examples of segmented images can be seen in figure 5. The white background is being classified as "fat". 


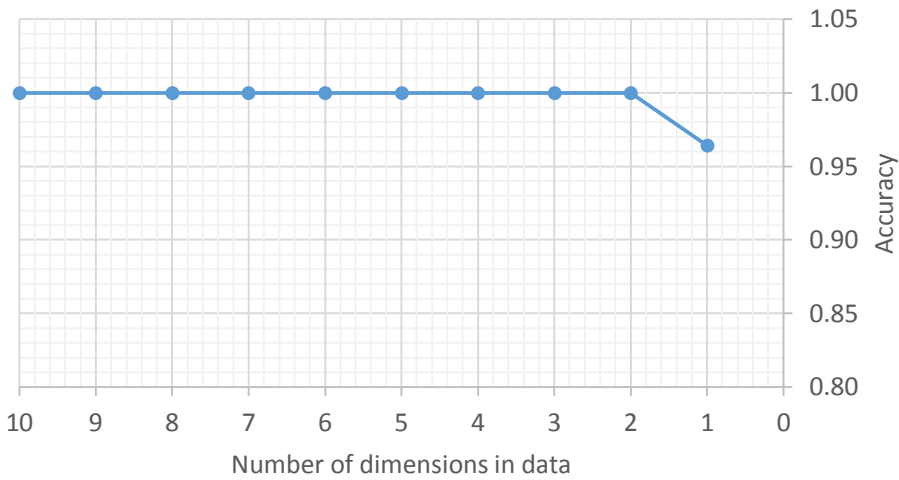

Figure 3: The accuracy of the decision tree as the dimensions are reduced. From 33 to 11 wavelengths are not depicted in the graph.

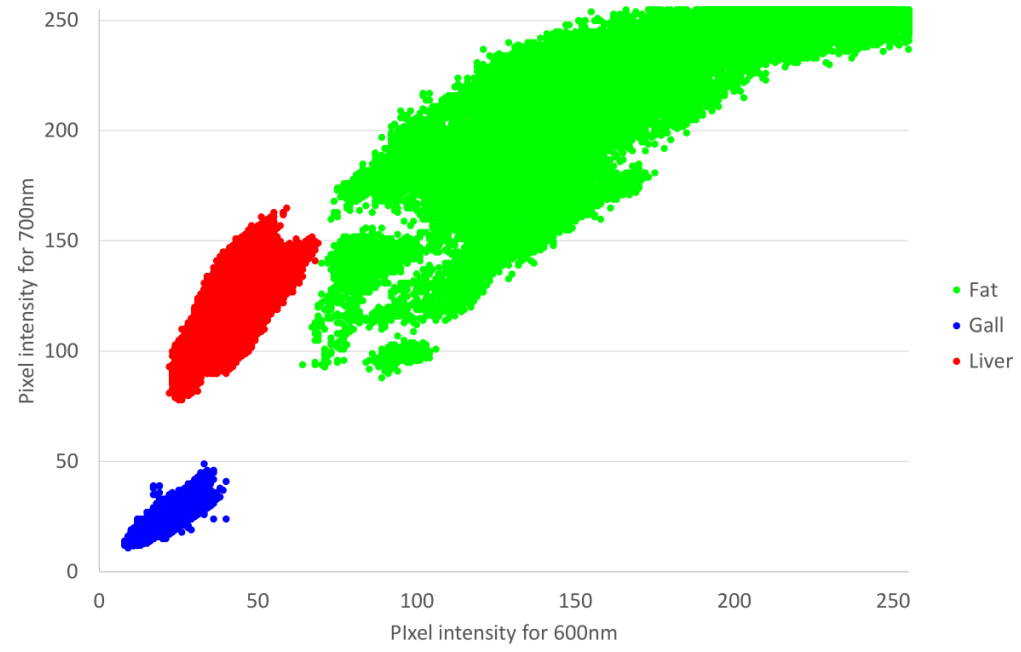

Figure 4: The intensity space using images captured at wavelength $600 \mathrm{~nm}$ and $720 \mathrm{~nm}$.

\subsection{Evaluate the images}

The data set should be classified into three groups. Images without a gallbladder("No Gall"), images with a gallbladder that can be removed("Good Gall") and images were the gallbladder can not be removed("Bad Gall"). In this study a gallbladder is considered removable if the entire contour is visible. A robot will in these situations be able to remove the gallbladder without puncturing it or damaging the liver.

Two features are extracted for this purpose. The first feature is the area of the gall in pixels. Blobs are created from the blue pixels in the segmented images. The area of the blobs is then summed, given the total area of the gallbladder in pixels. Holes inside the blobs are discarded, as these are either fat or reflections. Fat is not a problem for removal, as long as it is not connected to the liver.

The second feature is the ratio of the gall area divided by the area of the convex hull of all the gall blobs. In some segmented images the gall pixels are split into smaller blobs, often 


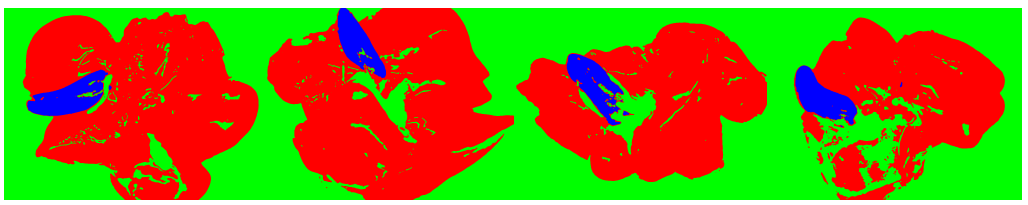

Figure 5: 4 examples of segmented images. Blue is gall, red is liver, and green is fat and background.

due to strings of fat on top of the gallbladder. All blobs in an image are therefore joined with the convex hull, to estimate the total area of the gallbladder. This can be seen in figure 6 .
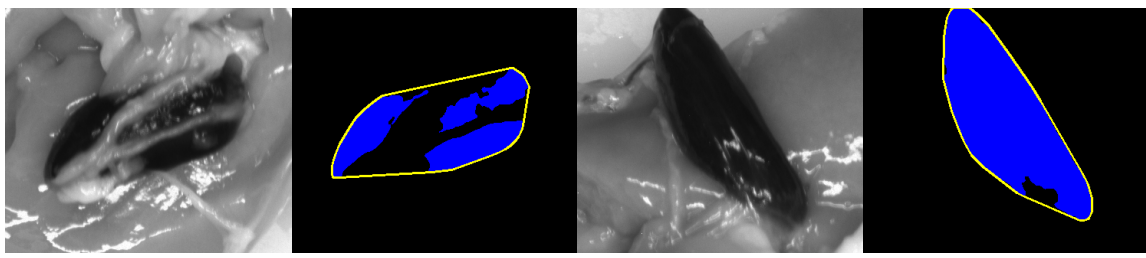

Figure 6: Finding the gallbladder in the images. The blue area describes the gall and the yellow line shows the convex hull around the gall areas.

\section{Results}

A classification tree has been trained using 10 fold cross validation, this time with a maximum of 4 nodes. The resulting confusion matrix can be seen in figure 7 . The overall accuracy of the system is $91.7 \%$. 89.5\% of "Bad Gall" were classified correctly, $72.7 \%$ of "Good Gall" were correct, and $100 \%$ "No Gall" were correctly classified.

\begin{tabular}{|c|c|c|c|c|c|}
\hline & \multicolumn{3}{|c|}{ Predicted Class } & \multirow[b]{2}{*}{ TPR/FNR } \\
\hline & & Bad Gall & Good Gall & No Gall & \\
\hline \multirow{5}{*}{ 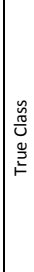 } & $\overline{\overline{\widetilde{O}}}$ & 17 & 2 & 0 & $89.5 \%$ \\
\hline & \begin{tabular}{l|}
$\vec{D}$ \\
$\mathbb{\infty}$ \\
$\infty$
\end{tabular} & $89.5 \%$ & $10.5 \%$ & $0.0 \%$ & $10.5 \%$ \\
\hline & $\begin{array}{l}\overline{\bar{त}} \\
\text { O } \\
\text { ס } \\
0\end{array}$ & $\begin{array}{c}3 \\
27.3 \%\end{array}$ & $\begin{array}{c}8 \\
72.7 \%\end{array}$ & $\begin{array}{c}0 \\
0.0 \%\end{array}$ & $\begin{array}{l}72.7 \% \\
27.3 \%\end{array}$ \\
\hline & $\overline{\overline{\widetilde{N}}}$ & 0 & 0 & 30 & $100.0 \%$ \\
\hline & शे & $0.0 \%$ & $0.0 \%$ & $100.0 \%$ & $0.0 \%$ \\
\hline
\end{tabular}

Figure 7: Confusion matrix showing the accuracy of the trained classifier.

\section{Discussion}

Of the small data set zero "No Gall" images were misclassified. So clean livers pass though the system as they should. Three "Good Gall" images were misclassified as "Bad Gall". 


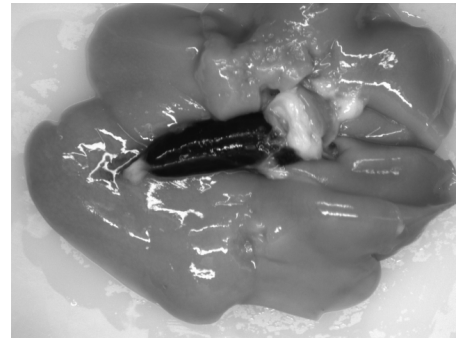

(a)

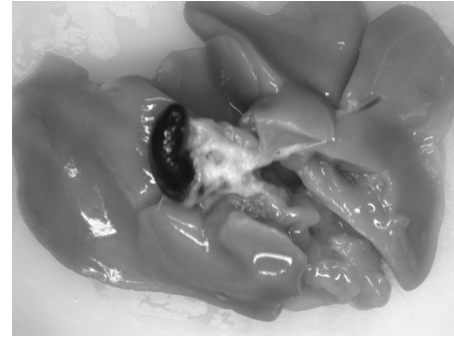

(b)

Figure 8: (a) This gallbladder is misclassified as "Good Gall". (b) This gallbladder is misclassified as "Bad Gall".

This would be expensive for a company as good livers are being sold as animal feed. Two "Bad Gall" images are predicted as "Good Gall". This error could also prove expensive if the gallbladder is punctured by the removal robot and ends up contaminating a whole batch of good livers.

Two error examples can be seen in figure 8. The gallbladder shown in figure 8(a) is wrongly classified as "Good Gall". The gall is almost completely free, but the "top" is covered with some liver and fat. The gallbladder shown in figure 8(b) is simply much smaller than the average and therefore misclassified as "Bad Gall".

The objects were segmented using images captured at $600 \mathrm{~nm}$ and $720 \mathrm{~nm}$. These are both in the upper end of the visible spectrum, especially $720 \mathrm{~nm}$ which also is the maximum of the used VariSpec filter. It would be interesting to extend the data set to include images captured at larger wavelengths, to investigate the information hidden outside the visible spectrum.

Overall, this preliminary study has shown that it is possible to detect the gallbladder on livers and, to some extend, tell whether they are removable or not. The gallbladder is easily separated from both the liver and fat using just one wavelength, yet two wavelengths are required for separating all three classes. Having two wavelengths also reduces the dependency of lighting conditions, especially if the segmentation is moved to colour space instead of intensity space. In general, the results looks promising but it should be tested on a larger data set before drawing conclusions.

\section{References}

[1] Kuanglin Chao, Chun Chieh Yang, and Moon S. Kim. Line-scan spectral imaging system for online poultry carcass inspection. Journal of Food Process Engineering, 34 (2011):125-143, 2011. ISSN 01458876.

[2] International Poultry Council. Chicken meat consumption for top producing countries. http://wWw. internationalpoultrycouncil.org/documents/ TopChickenConsumption.pdf, 2011.

[3] B. P. Dey, Y. R. Chen, C. Hsieh, and D. E. Chan. Detection of septicemia in chicken livers by spectroscopy,. Poultry Science, 82(2):199-206, February 2003. ISSN 00325791.

[4] Zheng Du, Myong K. Jeong, and Seong G. Kong. Band selection of hyperspectral im- 
ages for automatic detection of poultry skin tumors. IEEE Transactions on Automation Science and Engineering, 4(3):332-339, 2007. ISSN 15455955.

[5] Gamal Elmasry, Da Wen Sun, and Paul Allen. Near-infrared hyperspectral imaging for predicting colour, pH and tenderness of fresh beef. Journal of Food Engineering, 110 (1):127-140, 2012. ISSN 02608774.

[6] Hui Huang, Li Liu, and Michael O Ngadi. Recent developments in hyperspectral imaging for assessment of food quality and safety. Sensors (Basel, Switzerland), 14(4): 7248-76, 2014. ISSN 1424-8220.

[7] Mohammed Kamruzzaman, Gamal ElMasry, Da Wen Sun, and Paul Allen. Prediction of some quality attributes of lamb meat using near-infrared hyperspectral imaging and multivariate analysis. Analytica Chimica Acta, 714:57-67, 2012. ISSN 00032670.

[8] Yongyu Li, Jiajia Shan, Yankun Peng, and Xiaodong Gao. Nondestructive assessment of beef-marbling grade using hyperspectral imaging technology. ICAE 2011 Proceedings: 2011 International Conference on New Technology of Agricultural Engineering, pages 779-783, 2011.

[9] Linco. Classifeye. http://www. Iincofood. com/en/products/poultry_ processing/transfer_measurement/classifeye/index.html, 2015.

[10] Meyn. Quality grading system. https://www.meyn.com/en-GB/69/ quality-grading-system.html, 2015.

[11] Songyot Nakariyakul and David P. Casasent. Fast feature selection algorithm for poultry skin tumor detection in hyperspectral data. Journal of Food Engineering, 94(3-4): 358-365, 2009. ISSN 02608774.

[12] Efstathios Z. Panagou, Olga Papadopoulou, Jens Michael Carstensen, and George John E Nychas. Potential of multispectral imaging technology for rapid and nondestructive determination of the microbiological quality of beef filets during aerobic storage. International Journal of Food Microbiology, 174:1-11, 2014. ISSN 01681605.

[13] Bosoon Park, Seung Chul Yoon, William R. Windham, Kurt C. Lawrence, Moon S. Kim, and Kuanglin Chao. Line-scan hyperspectral imaging for real-time in-line poultry fecal detection. Sensing and Instrumentation for Food Quality and Safety, 5:25-32, 2011. ISSN 19327587.

[14] David G. Stork Richard O. Duda, Peter E. Hart. Pattern Classification. WileyInterscience, 2001.

[15] X rite Photo. Colorchecker classic. http://xritephoto.com/ph_product_ overview $\cdot$ aspx? ID=1192\&action=overview, 2015.

[16] Y Tao, J Shao, K Skeeles, and Y R Chen. Detection of splenomegaly in poultry carcasses by UV and color imaging. Transactions of the Asae, 43(2):469-474, 2000. ISSN 00012351.

[17] C-C Yang, K Chao, M S Kim, D E Chan, H L Early, and M Bell. Machine vision system for on-line wholesomeness inspection of poultry carcasses. Poultry science, 89: 1252-1264, 2010. ISSN 0032-5791. 
[18] Seung Chul Yoon, Bosoon Park, Kurt C. Lawrence, William R. Windham, and Gerald W. Heitschmidt. Line-scan hyperspectral imaging system for real-time inspection of poultry carcasses with fecal material and ingesta. Computers and Electronics in Agriculture, 79(2):159-168, 2011. ISSN 01681699. 